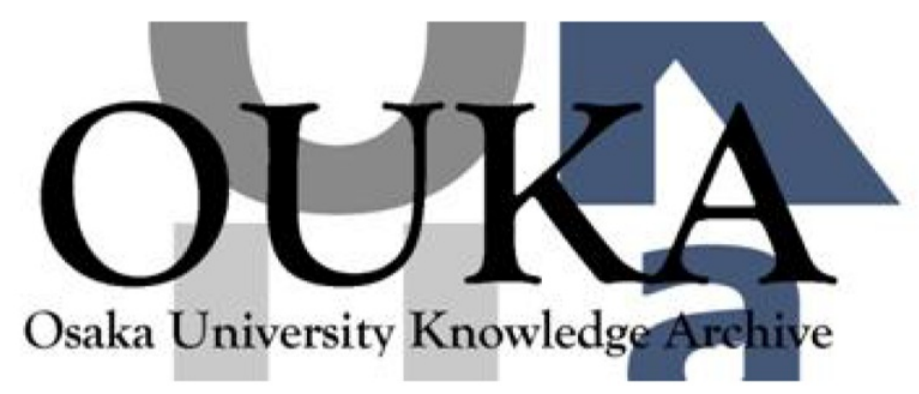

\begin{tabular}{|c|l|}
\hline Title & $\begin{array}{l}\text { Time-resolved optical and electrical study of } \\
\text { second-order processes responsible for the } \\
\text { formation of free polarons in conjugated } \\
\text { polymers }\end{array}$ \\
\hline Author(s) & $\begin{array}{l}\text { Hidayat, Rahmat; Nishihara, Yusuke; Fuji i, } \\
\text { Akihiko et al. }\end{array}$ \\
\hline Citation & Physical Review B. 66(7) p. 075214-p. 075214 \\
\hline Issue Date & $2002-08-30$ \\
\hline oaire:version & VoR \\
\hline URL & https://hdl.handle. net/11094/75674 \\
\hline rights & $\begin{array}{l}\text { Copyright (2002) by the American Physical } \\
\text { Society }\end{array}$ \\
\hline Note & \\
\hline
\end{tabular}

Osaka University Knowledge Archive : OUKA

https://ir. Library. osaka-u. ac. jp/

Osaka University 


\title{
Time-resolved optical and electrical study of second-order processes responsible for the formation of free polarons in conjugated polymers
}

\author{
Rahmat Hidayat, Yusuke Nishihara, Akihiko Fujii, Masanori Ozaki, and Katsumi Yoshino \\ Department of Electronic Engineering, Graduate School of Engineering, Osaka University, \\ 2-1 Yamada-oka, Suita, Osaka 565-0871, Japan \\ Eugene Frankevich \\ The Institute for Energy Problems of Chemical Physics, Russian Academy of Sciences, Moscow 117829, Russian Federation \\ and Department of Electronic Engineering, Graduate School of Engineering, Osaka University, \\ 2-1 Yamada-oka, Suita, Osaka 565-0871, Japan
}

(Received 24 March 2001; revised manuscript received 26 June 2001; published 30 August 2002)

\begin{abstract}
A technique based on the action of pairs of correlated laser light pulses (400 $\mathrm{nm}$ wavelength) with a controlled delay time between them was applied for studying second-order processes responsible for the formation of free charge carriers in a conjugated polymer poly(2,5-dioctyloxy- $p$-phenylenevinylene). Timeresolved experiments within the picosecond time domain enabled the attainment of direct evidence of the existence of intermediate states preceding free charge carriers at the photoexcitation of conjugated polymers. These states are shown to take part in second-order processes responsible for the generation of free charge carriers in conjugated polymers at a sufficiently high excitation density. The short lifetime of species involved in the processes led to the speculation that they are primarily formed polaron pairs, the majority of them being generated without any delay at the absorption of the light.
\end{abstract}

DOI: 10.1103/PhysRevB.66.075214 PACS number(s): 78.47.+p, 78.20.-e, 71.35. $-\mathrm{y}$, 71.20.Rv

\section{INTRODUCTION}

Photoconductivity of conjugated polymers is an important property that is useful in numerous applications of the materials. It is a complex physical phenomenon, depending on various fundamental factors, and is worth studying via various techniques. To date, two main approaches exist for describing photoconductivity; one of them considers absorbed light induced interband transitions, as in inorganic semiconductors, and the other treats the photoconductivity as a result of reactions with the participation of excited species formed under the light absorption (see review papers in Ref. 1). In this respect, the latter approach is similar to that in molecular crystals where excitons are primarily formed, and then their autoionization and electron-transfer reactions occur. However, recent studies carried out by the pump-and-probe technique have revealed that in contrast to molecular crystals, charge carriers appear within picoseconds after light absorption. ${ }^{2-5}$ In one work, ${ }^{3}$ such a conclusion was arrived at by measurement of the time-resolved formation of polaron pairs within the picosecond time domain by probing changes of the $\mathrm{cw}$ photocurrent instead of the light absorption. In connection with this fact, one has to note that the appearance of charged species in the material is a necessary but not sufficient condition for the $\mathrm{cw}$ photocurrent. The charged species must be free to drift under the action of an external electric field, and show a certain shift by the field during their lifetime. However, positive and negative charge carriers in molecular solids, being formed in pairs, remain in the mutual Coulomb field, and recombine geminately. ${ }^{6-9}$ This is a consequence of the low mobility of charge carriers or polarons. For geminately recombining polaron pairs, the net shift of charges is zero. Only some of the pairs manage to dissociate and contribute to the $\mathrm{cw}$ photocurrent. The thermally activated process of dissociation of polaron pairs ex- plains the temperature dependence of the $\mathrm{cw}$ photoconductivity. The latter becomes smaller on lowering the temperature, a typical value of the activation energy being about $0.2 \mathrm{eV}$.

Activated dissociation of polaron pairs does not appear to be the only way for free carrier formation. Our recent results (see Ref. 3) obtained on substituted polythiophene and poly ( $p$-phenylenevinylene) have shown that at laser pulse excitation, photoconductivity appears, which does not depend on the temperature. According to the model, primarily formed charge carriers belong to polaron pairs, and they can take part in the photoconductivity only if geminate recombination is prevented. These primarily formed polaron pairs contain polarons with higher (about $0.2 \mathrm{~cm}^{2} / \mathrm{V} \mathrm{s}$ ) and temperature independent mobility. ${ }^{3}$ At low temperatures, the pairs cannot dissociate, and it was conjectured that free polarons appear as a result of inter-pair recombination. Two charges from neighboring pairs recombine leaving two remaining charges at a larger intercharge distance thus increasing their chance to lie outside the Onsager radius and become free.

In the present work, we studied processes responsible for the formation of free polarons (free charge carriers) in samples of a typical conjugated polymer, $\operatorname{poly}(2,5-$ dioctyloxy- $p$-phenylenevinylene) (OO-PPV), under laser light (wavelength $400 \mathrm{~nm}$ ) excitation by a new technique based on the correlated action of two 150 fs laser pulses with a controlled delay time between them. There is a feature in the action which changes the yield of the products of the second-order processes as a function of the delay time under the condition of constant average intensity of laser excitation. The technique is selectively sensitive to the secondorder processes with the participation of intermediate active species having a lifetime within the range of the delay times used. It enables detection of those species and determination 
of their properties. Measurement of average photoconductivity induced by a sequence of pulses having a $1 \mathrm{kHz}$ repetition rate ensured taking into account free charge carriers only. The technique enabled the attainment of direct evidence on superlinear processes responsible for free polaron formation. The lifetime of intermediate states taking part in these processes is shown to be in the picosecond time domain. A possible scenario of the processes is suggested, which involves the primary formation of pretrapped polaron pairs, thermalization of the pairs, and interpair recombination.

\section{METHOD AND EXPERIMENT}

\section{A. Photoconductivity measurements}

In order to ensure that we are dealing with free charge carriers, it is necessary to measure the photoconductivity in the electrical circuit with an $R C$ value that is much higher than the lifetime of possible polarizable species formed under the action of light. In such a case, one should not be deterred by the displacement current of polarization nature, which may be responsible for the transient current measured in a low $R C$ circuit. ${ }^{10}$ In the present experiments, we measured the average photocurrent of OO-PPV excited by the 1 $\mathrm{kHz}$ sequence of $150 \mathrm{fs}$ laser pulses or pairs of the pulses with the wavelength of $400 \mathrm{~nm}$. dc voltage was applied to electrodes during the photoconductivity measurements.

\section{B. Two-pulse-correlation technique}

For studying the second-order processes responsible for the formation of free charge carriers, we suggested using a two-pulse-correlation laser technique. ${ }^{11}$ A pretext for looking for second-order processes was an observation of the superlinear dependence of the prompt component of the photoconductivity on the intensity of the laser pulses. ${ }^{3}$ In Ref. 11, we suggested splitting the pulses generated by the laser into pairs of pulses with controlled delay time between them and illuminating the sample by these correlated pulses. Although the total intensity of the pairs remained the same at any delay time, the yield of any second order processes would depend on the density of species taking part in the processes. The density itself depends on the superposition of the species produced by the first pulses of the pairs and surviving up to the moment of action of the second pulses. The rate of formation $U$ of products of a second-order process is believed to depend on the delay time $\Delta t$ and the intensity of light $I$ of two equal light pulses as

$$
U \propto I^{2}[1+f(\Delta t)] .
$$

Here, $f(\Delta t)$ is the function normalized to unity and describes the decay of species taking part in the second-order process. A prompt component of the transient photoconductivity recorded at a relatively high value of $R C$ or average photoconductivity may serve as a measure of the value of $U$. This formula appears as the result of integration of the products of a bimolecular reaction between intermediate species generated by two short pulses with intensities $I_{1}$ and $I_{2}$, the second one being delayed by $\Delta t$ in respect to the first one. In the case of $f(t)$ having an exponential view with $k_{1}$ as a monomolecular decay rate constant, the integration term is

$$
\begin{aligned}
U \propto & \int_{0}^{\Delta t}\left(I_{1} e^{-k_{1} t}\right)^{2} d t+\int_{\Delta t}^{\infty}\left(I_{1} e^{-k_{1}(t-\Delta t)}+I_{2} e^{-k_{1} t}\right)^{2} d t \\
& =I_{1}^{2}+I_{2}^{2}+2 I_{1} I_{2} e^{-k_{1} \Delta t} .
\end{aligned}
$$

In order to increase the sensitivity of the technique, we modulated the sequence of the first (undelayed) pulses by a chopper with a low frequency. A lock-in amplifier measured the $R$ vector and phase values of the modulated photocurrent at a low modulation frequency while changing the delay time. The modulated signal was proportional to the difference between signals produced by two sequences of pulses: undelayed and delayed pulses acting together and delayed pulses acting alone. The first sequence acts when the chopper (that modulates one beam only) is open, and the second acts when it is closed. One can show by simple calculations similar to those in Eq. (2.2), which include integration of all charge carriers formed, that the amplitude of the modulated rate of formation of free charge carriers, caused by bimolecular annihilation upon excitation by the sequence of two pulses $I_{1}$ and $I_{2}$, when the sequence of the first pulses is modulated, can be written as

$$
U \propto 1+2 \frac{I_{2}}{I_{1}} \exp \left(-k_{1} \Delta t\right) .
$$

Formulas (2.1) to (2.3) does not take into account the possible formation of products that are not connected with second-order reactions. The yield of those products will be modulated as well and therefore the ratio $R=U(\Delta t$ $=0) / U(\Delta t \rightarrow \infty)$ measured experimentally may be smaller than that resulting from formulas (2.1) to (2.3). A more exact description of the processes involved requires solving of the kinetic equation of the type used in this paper in Sec. IV.

Of course, second-order processes are revealed at a sufficiently high intensity of the light, which can be achieved by pulse laser light excitation. However, the technique permits us to obtain evidence on the very existence and properties of intermediate active species that work at any intensity of light, being precursors of the final products in first-order processes.

\section{Experimental setup}

In the present work, we studied a semiconducting polymer poly (2,5-dioctyloxy- $p$-phenylenevinylene) (OO-PPV). It was synthesized and purified by methods previously reported. ${ }^{12}$ Films of polymers were prepared by two techniques: (i) spin coating from chloroform solution (at a rotation speed of $300 \mathrm{rpm}$ ) and (ii) casting from toluene solution on the quartz substrates with comblike patterned interdigital $\mathrm{Au} / \mathrm{Cr}$ electrodes of $10 \mu \mathrm{m}$ width separated by a distance of 10 or $100 \mu \mathrm{m}$. The thickness of the polymer film was about a few $\mu \mathrm{m}$. The experimental setup is shown in Fig. 1. The electrical part was able to measure the modulated average photocurrent $\left(f_{\text {mod }}=20\right.$ to $\left.40 \mathrm{~Hz}\right)$ by the lock-in amplifier (Stanford Research System SR830) $(R C=0.01$ s). The tran- 


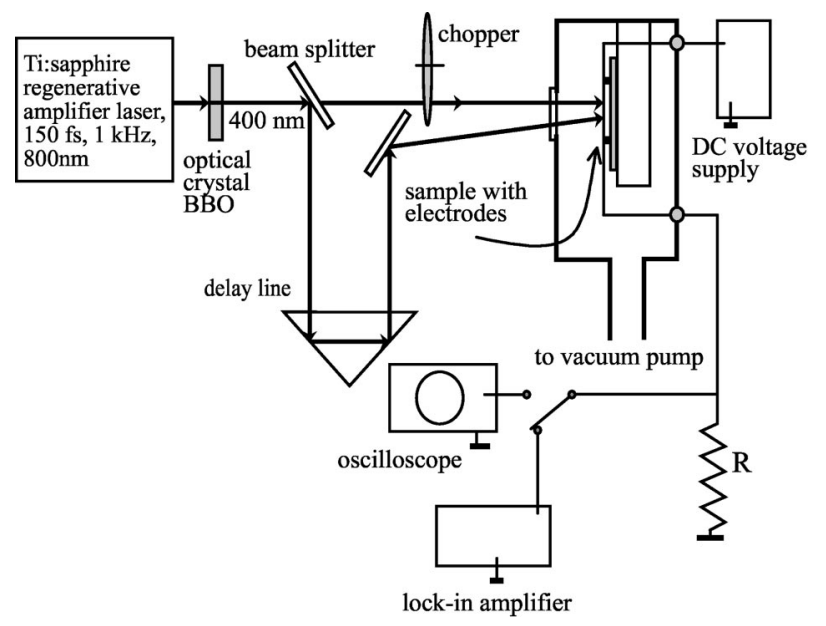

FIG. 1. Scheme of experimental setup. See the text for a description.

sient photocurrent was measured by the oscilloscope (Tektronix TDS $360-200 \mathrm{MHz}$ ) ( $R C$ about $1 \mu \mathrm{s}, 256$ pulses were accumulated). The sample was placed in the vacuum chamber $\left(10^{-5}\right.$ Torr $)$ at room temperature.

As the excitation source, a Ti:sapphire laser based regenerative amplifier system was used. The pulse width, wavelength and pulse repetition rate of the output laser beam were $150 \mathrm{fs}, 800 \mathrm{~nm}$, and $1 \mathrm{kHz}$, respectively. The beam were frequency-doubled by a 1-mm-thick BBO crystal to obtain a laser beam with the wavelength of $400 \mathrm{~nm}$. The beam was then divided into two parts by a beam splitter. Both parts were focused onto the sample. One beam was delayed using a computer controlled delay line. The delay time could be varied between $0.03 \mathrm{ps}$ and $1 \mathrm{~ns}$. Pulse energies were measured, and the light intensity at the sample position could be varied within a range from 1 to $20 \mu \mathrm{J}$ per pulse. The illumination area on the sample was about $0.04 \mathrm{~cm}^{2}$.

\section{EXPERIMENTAL RESULTS}

We measured the photocurrent induced in the electric circuit under excitation of a sample by laser pulses in vacuum at room temperature. Typical transient photocurrent decays of an OO-PPV sample are presented in Fig. 2. We used OOPPV samples prepared by spin coating from chloroform solution. The decay has a prompt component at the point of application of excitation pulses, a decaying part that is due to the final $R C$ value of the electrical circuit (about $1 \mu \mathrm{s}$ ), and a delayed part at longer time periods. A superlinear dependence of the amplitude of the photocurrent at $t=0$ on the light intensity was detected previously as reported in Ref. 3. Measurements in the two-correlated-pulse regime have shown that the prompt component changed when excited at the delay time $\Delta t$ greater than zero. This can be seen in Fig. 2 , where higher and lower curves were measured at the same intensity of excitation light at $\Delta t=0$ and $\Delta t>10 \mathrm{ps}$, respectively. Figure 3 shows the dependence of the peak of the transient curves of the photocurrent on the delay time. A peak centered at zero delay time was observed with the halfwidth of about $1.6 \mathrm{ps}$ at $1 / e$ of the maximum intensity. In

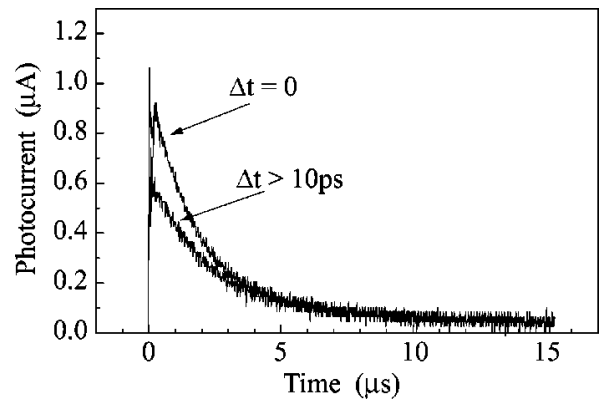

FIG. 2. Photocurrent induced by pairs of correlated light pulses measured by an oscilloscope at $\Delta t>10$ ps (lower curve), and when the pulses overlapped at $\Delta t=0$ (upper curve). The sample is a film of OO-PPV prepared by spin coating from chloroform solution. The light pulse power is $9 \mu \mathrm{J} /$ pulse; $R C=1 \mu \mathrm{s}$; the gap between electrodes $=100 \mu \mathrm{m}$, and $V=300 \mathrm{~V}(E=30 \mathrm{kV} / \mathrm{cm})$.

addition to this $1.6 \mathrm{ps}$ half-width narrow peak, a broader one with a half width of about 5 ps that is much weaker in intensity was also observed.

Figure 4(a) presents similar results obtained for an OOPPV sample measured by the lock-in amplifier with the first pulse modulation. The results were obtained for a sample prepared by casting from toluene solution and have allowed us to gain more information. The modulated average photocurrent was measured from zero delay time up to a delay time $\Delta t=1 \mathrm{~ns}$ at different intensities of the light pulses as indicated in the figure. The inset of Fig. 4(a) shows the dependence of the maximum average photocurrent (at zero delay time) on the intensity of the light pulses that becomes superlinear at a rate higher than $4 \mu \mathrm{J} /$ pulse. Figure 5 presents the corresponding peaks within a shorter delay time range, $\Delta t=150$ ps. It is evident that the width of the peaks does not depend on the intensity of the light, and the half-width at $1 / e$ of the maximum intensity is about $14 \mathrm{ps}$.

The ratio $R$ of the photocurrents $i_{\mathrm{ph}}$ at $\Delta t=0$ ps and $\Delta t$ $=500$ ps was measured to be about 1.5 which shows that bimolecular processes are the main providers of free charge carriers at the intensities used.

We also measured the kinetics of fluorescence decay of an

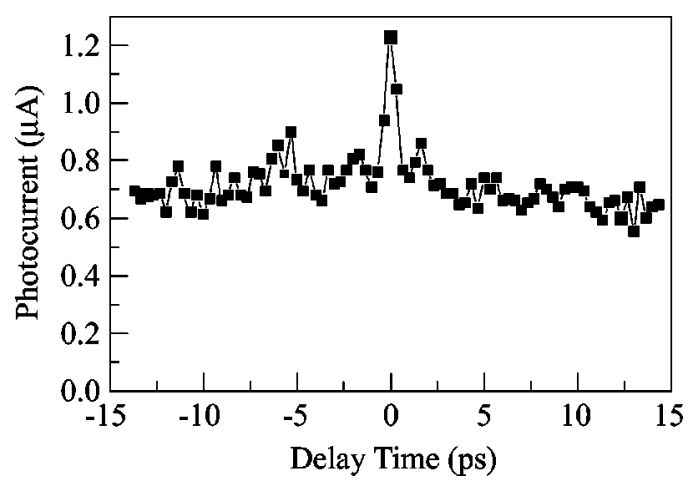

FIG. 3. Amplitude of the photocurrent measured by an oscilloscope as a function of delay time $\Delta t$ for the same sample as in Fig. 2. The light pulse power is $9 \mu \mathrm{J} /$ pulse, and $V=300 \mathrm{~V}(30 \mathrm{kV} / \mathrm{cm})$. The curve seems to be composed of two components with half widths at $1 / e$ of a height of about 1.6 and 5 ps. 

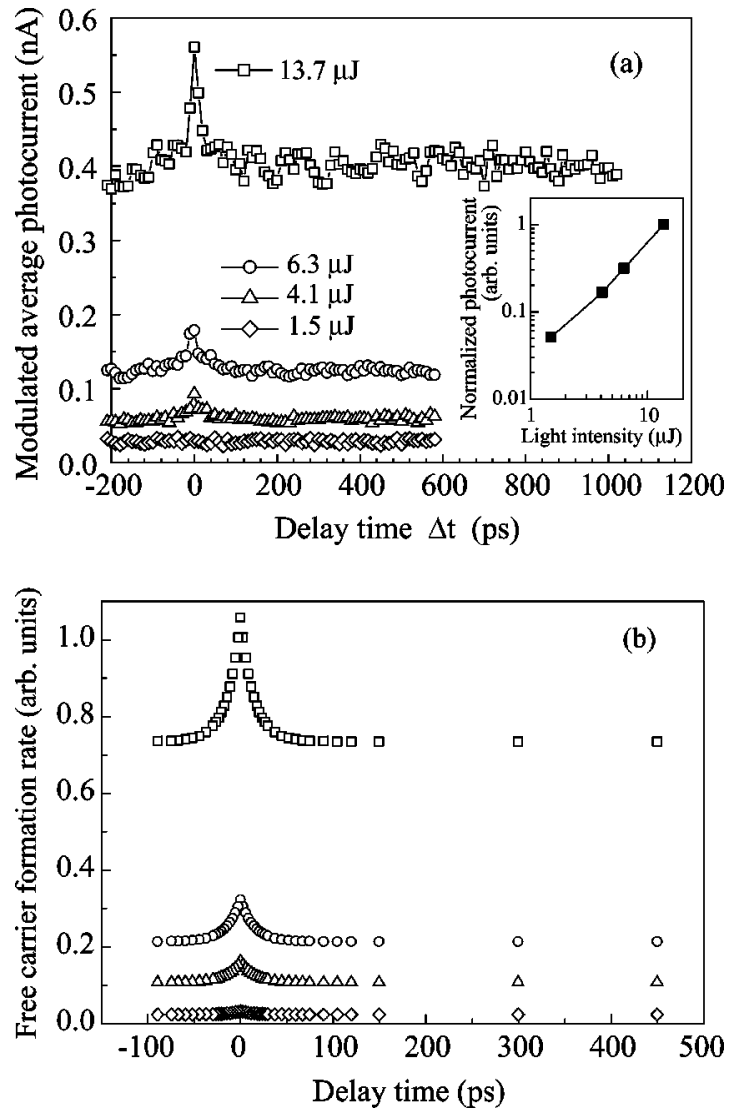

FIG. 4. Investigation of the samples of OO-PPV, prepared by casting from toluene solution, by the first-pulse-chopped modulation technique. (a) Experimental dependencies of the modulated average photocurrent, measured by a lock-in amplifier at the modulation frequency equal to $20 \mathrm{~Hz}$, on the delay time $\Delta t$ at different light pulse intensities $13.7 \mu \mathrm{J} /$ pulse, $6.3 \mu \mathrm{J} /$ pulse, $4.1 \mu \mathrm{J} / \mathrm{pulse}$, and $1.5 \mu \mathrm{J} /$ pulse for upper to lower curves, correspondingly. The gap between electrodes $=10 \mu \mathrm{m}$, and $V=18 \quad \mathrm{~V}(E=18 \mathrm{kV} / \mathrm{cm})$. Inset: The peak of the modulated average photocurrent at $\Delta t=0$ plotted as a function of the light intensity. (b) The same as (a) but computer calculated by Eqs. (4.1) and (4.2), see text for details.

OO-PPV film using a streak camera. The measurement, however, was performed using low-intensity light excitation, namely, a 90 fs pulse laser with an energy of a few $\mathrm{pJ} /$ pulse. The laser wavelength was $400 \mathrm{~nm}$. The decay is shown in Fig. 6, indicating the lifetime of singlet exciton $\tau_{\text {exc }}$ of about 400 ps.

\section{DISCUSSION}

The present results demonstrate that second-order processes are responsible for the generation of free polarons at high intensity light excitation within the range of 1.5 to 13.7 $\mu \mathrm{J} /$ pulse, which corresponds to the range of photon absorption rates from $3.6 \times 10^{18}$ to $3.3 \times 10^{19}$ photon $/ \mathrm{cm}^{3}$ per pulse (if the extinction coefficient $\varepsilon=5 \times 10^{4}$ is applied). The halfwidth of the peaks in Figs. 3, 4, and 5, which lie within the ps time domain, yields the lifetimes of excited species taking part in the second order processes.

One can think of a few processes that are formally able to

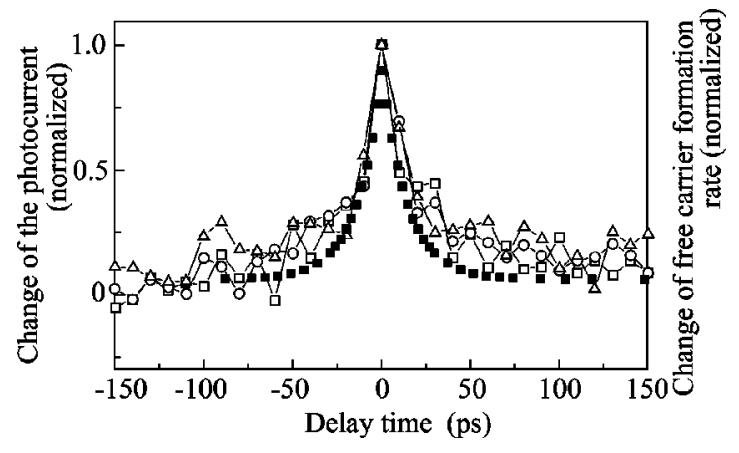

FIG. 5. Modulated average photocurrent in Fig. 4(a) for light intensities of $4.1 \mu \mathrm{J} /$ pulse and $13.7 \mu \mathrm{J} /$ pulse redrawn in the normalized peak intensity and extended scale of the delay time. Open points are for experimental data, solid squares are for results of computer calculations based on Eqs. (4.1) and (4.2). The half-width of the curves at $1 / e$ of the height equals $14 \mathrm{ps}$.

account for the superlinear dependence of the photoconductivity on the light intensity. We consider the following.

(1) Photons deal with intrachain excitons $A^{*}$ and produce free polarons $P^{+}$and $P^{-}$:

$$
A^{*}+h \nu \rightarrow P^{+}+P^{-}
$$

(2) Intrachain exciton $A^{*}$ interacts with one charge of a polaron pair $P^{+} \ldots P^{-}$and causes dissociation of the pair

$$
\left(P^{+} \ldots P^{-}\right)+A^{*} \rightarrow P^{+}+P^{-} .
$$

A similar second-order reaction, which leads to the formation of a free polaron, is an interaction of $A^{*}$ with a trapped charge carrier

$$
A^{*}+P_{\text {trapped }}^{+}\left(\text {or } P_{\text {trapped }}^{-}\right) \rightarrow P^{+}\left(\text {or } P^{-}\right) .
$$

(3) Two intrachain excitons interact with each other accumulating in a sufficient single-site energy for the formation of free charge carriers

$$
A^{*}+A^{*} \rightarrow P^{+}+P^{-} \text {. }
$$

(4) A photon excites a charge carrier within the polaron pair that enables it to escape from the pair

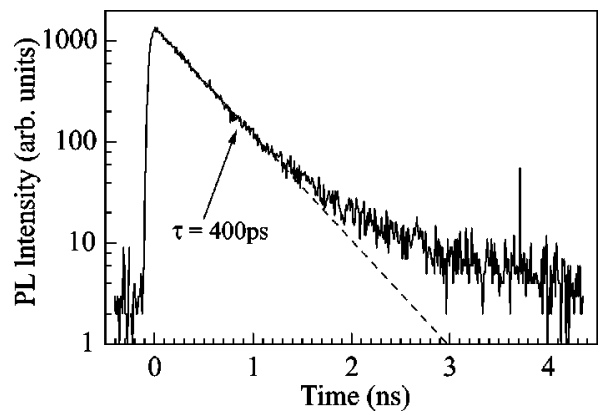

FIG. 6. Photoluminescence decay of an OO-PPV film excited by a $90 \mathrm{fs}$ width laser pulse with the wavelength of $400 \mathrm{~nm}$, and a weak light intensity of a few pJ. Initial decay gives the value of the lifetime of intra-chain excitons in OO-PPV equal to $\approx 400$ ps. 


$$
\left(P^{+} \ldots P^{-}\right)+h \nu \rightarrow P^{+}+P^{-} .
$$

(5) Two photons acting together excite the system to a high sufficiently level

$$
h \nu+h \nu \rightarrow P^{+}+P^{-} .
$$

(6) Interpair recombination: It takes place when a newly generated pair appears near adjacent to a pair generated earlier. Two neighboring polarons recombine causing two others to be free:

$$
\left(P^{+} \ldots P^{-}\right)+\left(P^{+} \ldots P^{-}\right) \rightarrow P^{+}+P^{-} .
$$

The problem is how to choose a proper process on the basis of experimental results. The results yield the lifetime of species involved in the process. Intrachain excitons working in process (1) can be excluded when they have the lifetime $\tau_{\text {exc }}$ much longer than a few ps, namely, about 400 ps as evident in Fig. 6. Special attention has to be paid to processes (2) involving intrachain excitons because they, being formed initially, contain excess vibration energy and may be considered as having a much higher reactivity than the relaxed ones though only for a short time. One could assume then that those highly reactive ("hot") excitons show themselves in a second-order process producing free charge carriers either by causing dissociation of polaron pairs or just by detrapping charge carriers residing in some traps. Moreover, a probability remains that intra-chain excitons preserve a high activity for a longer time within a ps time domain during their relaxation within the inhomogeneously broadened density of states. So, formally, we may consider process (2) as a candidate, responsible for at least a part of the secondorder processes observed. However, we remain sceptical about the important role played by this process. This is because of our experience (see our paper ${ }^{3}$ ) that excitation of a charge in a polaron pair results in a very small change of the probability of its dissociation (which may be positive or negative) connected with an increased mobility of the charge. This leads to increased rates of both dissociation, and geminate recombination of polaron pairs. One more point concerns the reactivity of intrachain excitons after their relaxation. It is known that they retain the ability to react with charge carriers despite having an internal energy lower than the initial one. Even triplet excitons having energy about 1 $\mathrm{eV}$ lower are able to detrap charge carriers responsible for the well-known photoenhanced current. As long as Figs. 3 to 5 do not show the involvement of species with lifetimes of relaxed (400 ps) excitons we may assume that process (2) is not important for the conditions considered by us.

It has been much emphasized that processes involving the participation of intrachain excitons can take part in the formation of free polarons. The work in Ref. 13 suggests process (3) is partially responsible for the formation of polaron pairs and charge carriers. However, they are not visible at delay times within the picosecond time domain as long as the excitons have a longer lifetime. Process (4) deals with excitation of charge carriers within a pair, and it can be expected that their behavior is similar to that previously reported for the action of red light pulses on photocurrent, ${ }^{3}$ i.e., the in- crease or decrease of the photocurrent depending on the intensity of the light. The narrow peaks in Figs. 3, 4, and 5 require that both species involved in the second-order process should be short lived. Process (4) is assumed to be effective for thermalized polaron pairs. And if the lifetime of those pairs is sufficiently short and polarons are able to absorb the light of $400 \mathrm{~nm}$ wavelength then process (4) might be considered favorably though its effectiveness is expected to be low. ${ }^{3}$ Moreover, a fast decay during the delay times of the order of the laser pulse width (150 fs) would be expected for this process. However, it is absent. Process (5) may occur if only two laser pulses overlap, and a peak can be expected in the dependence of the photocurrent on the delay time with the peak width of a few hundreds of fs. The observed peaks are broader. Thus, we arrive at the conclusion that process (6) may be responsible for the formation of free polarons from polaron pairs. Recently, we succeeded in observing an effect of the electrical field (within the range up to 2 $\times 10^{5} \mathrm{~V} / \mathrm{cm}$ ) on the dissociation of the species involved in the second-order processes. ${ }^{16}$ These latter results support the conclusion.

The results obtained previously ${ }^{3}$ have permitted us to assume the existence of two types of polaron pairs. Primarily, polaron pairs $P_{1}$ are produced in a highly mobile state [mobility of primarily formed polarons was estimated as 0.2 $\mathrm{cm}^{2} / \mathrm{V} \mathrm{s}$ (Ref. 14)]. However, after fast thermalization, the pairs become trapped pairs $P_{2}$, i.e., polarons become localized on conjugated segments of polymer molecules and their mobility drops by 3 to 4 orders of magnitude. At a high intensity of light excitation, inter-pair recombination can be expected as one method of forming free charge carriers although it occurs at the expense of a loss of the partners in the pairs.

In the present work, the measurements within the range of time delay up to 1 ns show two values of peak width, which certainly originated from second-order processes of excited states with different lifetimes. The lifetime of pretrapped polaron pairs estimated in Refs. 3 and 15 is expected to be about a few ps, and that of thermalized polaron pairs is expected to be of a higher value. Two values of the lifetime seen in Fig. 3 may be connected with those pairs. But one can assume that polaron pairs that show themselves in more detailed experiments conducted on the OO-PPV samples obtained by a casting technique (Figs. 4 and 5) correspond to thermalized polaron pairs.

It should be noted that the width of the peak appeared to be dependent on the method of preparation of the sample. It was much narrower for the samples prepared by a spin coating technique. One can assume that it is a sequence of different structures of the polymer film, which affects the thermalization rate or /and mobility of polarons in the pairs. This feature deserves to be studied in more detail. In the present work we paid more attention to samples prepared by the casting technique.

The kinetic equation that would be able to describe the formation and recombination of polaron pairs $P_{2}$ can be written as 


$$
\frac{d P_{2}}{d t}=g(t)-\left(k_{g 2}+k_{t h}\right) P_{2}-k_{22} P_{2}^{2},
$$

where at $t=0 P_{2}=0$. Here, $g(t)$ is the rate of formation of polaron pairs. In the two-correlated-pulse regime, $g(t)$ $=g_{1} e^{-\left[(t-\Delta t) / t_{0}\right]^{2}}+g_{2} e^{-\left(t / t_{0}\right)^{2}}$, where $t_{0}$ is the width of the pulse, $\Delta t$ is the delay time, and $P_{2}$ is the density of thermalized polaron pairs. The constant $k_{g 2}$ is the rate constant for geminate recombination, $k_{22}$ is the rate constant for random recombination (interpair recombination), and $k_{\mathrm{th}}$ is the rate constant for the dissociation of polaron pairs and the formation of free polarons. Here, we assumed that the pairs appeared immediately under the action of the light or they were formed as a result of sufficiently fast thermalization of pretrapped polaron pairs. However, we also examined how the inclusion of delayed formation of the pairs from intrachain excitons affects the final results.

Two terms in Eq. (4.1) may be considered as being responsible for the appearance of free charge carriers. They are (i) the term $k_{22} P_{2}^{2}$ (interpair random recombination of two charges from different pairs leaves two other charges at a greater intercharge distance and gives them a chance to get free) and (ii) the term $k_{\mathrm{th}} P_{2}$ describing thermal- and electrical-field-assisted dissociation of polaron pairs.

The next kinetic equation, which works for the accumulation of free polarons $p$ after the action of two correlated pulses, can be written as

$$
\frac{d p}{d t}=\beta_{1} k_{22} P_{2}^{2}+k_{t h} P_{2},
$$

where at $t=0 p=0 ; \beta_{1}<1$ is the yield of free charge carriers formed per interpair recombination event. The solution of the system of Eqs. (4.1) and (4.2), which involves the integration of Eq. (4.2) over the period much longer than lifetimes of the species taking part in the second order processes, enables us to obtain the yield of free charge carriers formed under photoexcitation that is directly proportional to the value of $p$ obtained in that way. We assume that the photocurrent measured experimentally is directly proportional to $p$.

It should be noted that the kinetic equation on for polaron pairs in the form of Eq. (4.1) contains a few approximations. We treat polaron pairs here as quasiparticles able to interact with each other as if they were movable. This permits us to introduce a kinetic rate constant for interpair recombination. However, it is quite possible that polaron pairs interact with each other not due to their random motion but because of an accidental mutual superposition at the instant of their formation. Formally it means that rate constant $k_{22}$ is connected with the effective volume occupied by a pair $\Delta V_{0}$ and the light pulse width $t_{0}$, rather than with the diffusion of the pairs $\left(k_{22} \approx \Delta V_{0} / t_{0}=6.7 \times 10^{-9} \mathrm{~cm}^{3} / \mathrm{s}\right.$ at a reasonable value of $\Delta V_{0}=10^{-21} \mathrm{~cm}^{3}$ and $t_{0}=150 \mathrm{fs}$ ). By describing the polaron pair as quasiparticles we are making one more simplification: geminate recombination though being a firstorder process obeys the kinetics that differs from exponential decay. Polaron pairs survive for the time interval required for polarons to cross the intercharge distance, and only after that do they disappear. Thus, the lifetimes of polaron pairs measured here describe, within the framework of this model, not the disappearance of polaron pairs but, rather, the kinetics of decrease of the intercharge distance in the pairs, which is responsible for the values of $\Delta V_{0}$ and $k_{22}$.

We solved the system of Eqs. (4.1) and (4.2) by computer simulation and arrived at the dependencies shown in Figs. 4(b) and 5. The values of the rate constants were regulated in order to fit the general features of experimental curves. The most important parameters in the experimental curves, which appeared to be important for choosing the rate constants, were (a) the half width of the peak connected with the lifetime of the species taking part in a second-order process and (b) the maximum relative change of the photocurrent that corresponds to an increase of the delay time from zero to a much longer value. The last change was equal to 1.5 as can be seen in Fig. 4(a). The values of the rate constants were calculated on the basis of these parameters $k_{g 2}+k_{t h}=1 / \tau_{p}$ $=6.7 \times 10^{10} \mathrm{~s}^{-1}$ [it is the experimental value obtained from the half-width of the peak in Fig. 4(a)], $k_{22}=4$ $\times 10^{-9} \mathrm{~cm}^{3} / \mathrm{s}$ (but it was revealed that the results were not sensitive to values of $k_{22}$ if the latter are smaller than the indicated value). The maximum relative change of the photocurrent with the delay time was revealed to be very critical for choosing the ratio $S$ of probabilities of formation of free carriers from a recombination event and from the dissociation of polaron pairs $S=\beta_{1} / k_{\mathrm{th}} \tau_{p}$. The ratio was calculated as $S=20$, and on the basis of this we have taken arbitrarily $\beta_{1}=0.1$ and $k_{\mathrm{th}}=3.3 \times 10^{8} \mathrm{~s}^{-1}$. Calculations were performed for polaron pair generation rates $g_{1}=g_{2}$ from 4.8 $\times 10^{30}$ to $4.4 \times 10^{31} \mathrm{~cm}^{-3} \mathrm{~s}^{-1}$ corresponding to light pulse power from 1.5 to $13.7 \mu \mathrm{J} /$ pulse under the assumption of the quantum yield of polaron pair formation to be 0.2 and extinction coefficient to be $5 \times 10^{4} \mathrm{~cm}^{-1}$. Figure 4(b) shows the dependencies of the free carrier formation rate on delay time calculated for the same light intensity as measured in the experimental work. The calculated changes of photocurrent as a function of light intensity satisfactorily fit the experimental result as shown in Fig. 4(a). The peak having the half-width of about $14 \mathrm{ps}$ corresponds to that observed in the experimental work as well, which is shown by solid squares in Fig. 5 together with the normalized experimental results.

In our calculations, we assumed that polaron pairs were formed without any delay under the action of light. Such a viewpoint was supported by the results of the works performed using the pump-and-probe technique ${ }^{2,4,5}$ and by those of our experiments. ${ }^{3,15} \mathrm{We}$ tried, nevertheless, to add a new term to Eq. (4.1) of the type $g \exp \left(-t / \tau_{\text {exc }}\right)$, with $\tau_{\text {exc }}$ $=400 \mathrm{ps}$, which would correspond to the delayed formation of polaron pairs from relaxed intra-chain excitons. The effect of the delay time $\Delta t$ on the formation of the excitons was taken into account. In performing the calculations, we maintained the total amount of polaron pairs formed from dissociation of excitons $N_{\text {from exciton }}$ and formed directly $N_{\text {direct }}$ : $N_{\text {from exciton }}+N_{\text {direct }}=$ const, but varied the ratio $N_{\text {from exciton }} / N_{\text {direct }}$ from 0 to 10 . An addition of the terms describing the generation of polaron pairs from excitons resulted in appearance of a noticeable tail in the calculated dependence of the photocurrent vs delay time at $\Delta t$ up to 


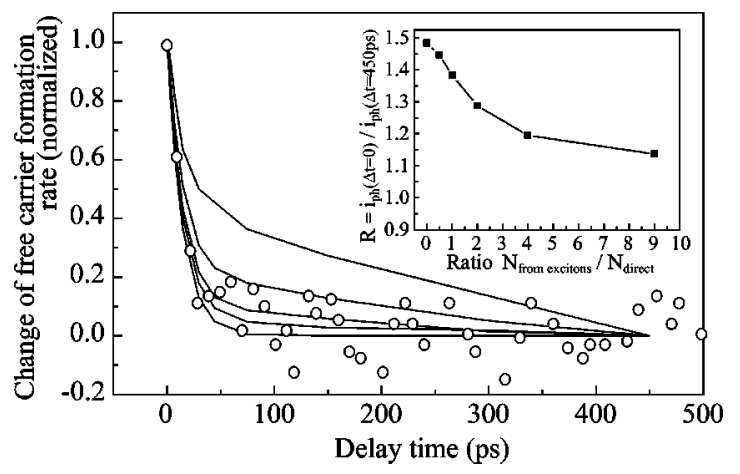

FIG. 7. Effect of addition of an assumed process of the polaron pair formation by dissociation of molecular excitons. Solid lines were calculated by solving the system of Eqs. (4.1) and (4.2) with the addition of the delayed generation term to Eqs. (4.1). Total amount of the polaron pairs was kept constant, but the ratio $N_{\text {from excitons }} / N_{\text {direct }}$ was varied from 0 to 10 . The higher to lower curves shown correspond to the ratios equal to $4,2,1,0.5$, and 0 , respectively. The inset in the figure shows the calculated ratio $R$ of maximal photocurrent (at $\Delta t=0$ ) to the minimal one (at $\Delta t$ $=500 \mathrm{ps}$ ) as a function of the relative amount of pairs formed by exciton dissociation. Open circles are normalized experimental data taken from Fig. 4(a), upper curve. One can see that experimental ratio $R=1.5$ and a reasonable tail can be obtained under the condition that the main part of the pairs is formed immediately under the action of the light pulse $N_{\text {from excitons }} / N_{\text {direct }} \leqslant 1$.

about 400 ps at $N_{\text {from excitons }} / N_{\text {direct }}>1$, as is shown in Fig. 7. The curves were calculated by using parameters corresponding to the upper curves in Figs. 4(a) and 4(b). The tail is not visible in the experiment as can be seen from the comparison of experimental (open circles) and calculated dependence presented in Fig. 7. Moreover, inclusion of the delayed generation of polaron pairs led to a decrease of the ratio $R$ $=i_{\mathrm{ph}}(\Delta t=0) / i_{\mathrm{ph}}(\Delta t=450 \mathrm{ps})$ calculated, as is shown in the inset of Fig. 7. Satisfactory fitting of experimental data with $R=1.5$ could be preserved if it was considered that not less than one half of polaron pairs were formed directly under the action of the light or, possibly, as a result of a fast reaction of primarily formed "hot" intrachain excitons. The rest may be considered to be produced as a result of a charge transfer from intrachain excitons during their total lifetime.

\section{CONCLUSION}

The technique based on the action of pairs of correlated light pulses with a controlled delay time between them was applied successfully to studying second-order processes responsible for the formation of free charge carriers in conjugated polymers. We worked with poly(2,5-dioctyloxy$p$-phenylenevinylene), performed time-resolved experiments within the picosecond time domain, and obtained direct evidence on the existence of intermediate states preceding free charge carriers at the photo excitation of conjugated polymers. They are shown to take part in second-order processes responsible for the generation of free charge carriers in conjugated polymers at a high excitation density. The short lifetime of species involved in the processes permitted us to speculate that those are primarily formed polaron pairs, their main part being generated without any delay in the absorption of light.

\section{ACKNOWLEDGMENTS}

E.F. is indebted to JSPS for the support of his stay in Japan. The work was supported partially by Grant INTAS No. 97-0992 and Grant RFBR No. 00-03-32253. The work was also supported by a Grant-in-Aid for Scientific Research from Ministry of Education, Culture, Sports, Science and Technology of Japan.
${ }^{1}$ Primary Photoexcitations in Conjugated Polymers: Molecular Excitons vs. Semiconductor Band Model, edited by N.S. Sariciftci (World Scientific, Singapore, 1997).

${ }^{2}$ D. McBranch, A. Hays, M. Sinclair, D. Moses, and A.J. Heeger, Phys. Rev. B 42, 3011 (1990).

${ }^{3}$ E. Frankevich, H. Ishii, Y. Hamanaka, T. Yokoyama, A. Fujii, S. Li, K. Yoshino, A. Nakamura, and K. Seki, Phys. Rev. B 62, 2505 (2000).

${ }^{4}$ G. Cerullo, S. Stagira, M. Nisoli, S. De Silvestri, G. Lanzani, G. Kranzelbinder, W. Graupner, and G. Leising, Phys. Rev. B 57, 12806 (1998).

${ }^{5}$ D. Moses, A. Dogariu, and A.J. Heeger, Chem. Phys. Lett. 316, 356 (2000).

${ }^{6}$ E.L. Frankevich, A.A. Lymarev I. Sokolik, F.E. Karasz, S. Blumstengel, R.H. Baughman, and H.H. Hörhold, Phys. Rev. B 46, 9320 (1992).

${ }^{7}$ E.M. Conwell and H.A. Mizes, Phys. Rev. B 51, 6953 (1995).
${ }^{8}$ S. Barth and H. Bassler, Phys. Rev. Lett. 79, 4445 (1997).

${ }^{9}$ M. Yan, L.J. Rothberg, F. Papadimitrakopoulos, M.E. Galvin, and T.M. Miller, Phys. Rev. Lett. 72, 1104 (1994).

${ }^{10}$ E. Frankevich, Chem. Phys. Rep. 17, 2069 (1999).

${ }^{11}$ E. Frankevich, R. Hidayat, A. Fujii, M. Ozaki, and K. Yoshino, J. Soc. Electrical Mater. Eng. 9, 109 (2000).

${ }^{12}$ G.J. Sarnecki, P.L. Burn, A. Kraft, R.H. Friend, and A.B. Holmes, Synth. Met. 55-57, 914 (1993).

${ }^{13}$ V.I. Klimov, D.W. McBranch, N.N. Barashkov, and J.P. Ferraris, Chem. Phys. Lett. 277, 109 (1997).

${ }^{14}$ D. Moses, J. Wang, G. Yu, and A.J. Heeger, Phys. Rev. Lett. 80, 2685 (1998).

${ }^{15}$ E. Frankevich, H. Ishii, Y. Hamanaka, T. Yokoyama, A. Fujii, S. Li , K. Yoshino, A. Nakamura, and K. Seki, Synth. Met. 119, 495 (2001).

${ }^{16}$ E. Frankevich, Y. Nishihara, A. Fujii, M. Ozaki, and K. Yoshino, Phys. Rev. B (to be published). 